\title{
Sequence Comparison of Vaginolysin from Different Gardnerella Species
}

\author{
Erin M. Garcia ${ }^{1,+}$, Myrna G. Serrano ${ }^{1,2,+}{ }^{\text {, Laahirie Edupuganti }}{ }^{1,2}$, David J. Edwards ${ }^{2,3}$, Gregory A. Buck ${ }^{1,2,4}$ \\ and Kimberly K. Jefferson ${ }^{1,2, *}$
}

1 Department of Microbiology and Immunology, Virginia Commonwealth University, Richmond, VA 23298, USA; egarcia26@wisc.edu (E.M.G.); myrna.serrano@vcuhealth.org (M.G.S.); Laahirie.edupuganti@vcuhealth.org (L.E.); gregory.buck@vcuhealth.org (G.A.B.)

2 Center for Microbiome Engineering and Data Analysis, Virginia Commonwealth University, Richmond, VA 23298, USA; dedwards7@vcu.edu

3 Department of Statistical Sciences and Operations Research, Virginia Commonwealth University, Richmond, VA 23298, USA

4 Department of Computer Science, College of Engineering, Virginia Commonwealth University, Richmond, VA 23298, USA

* Correspondence: Kimberly.jefferson@vcuhealth.org; Tel.: +01-804-828-9699

+ The authors contributed equally as co-first authors.

Citation: Garcia, E.M.; Serrano, M.G.; Edupuganti, L.; Edwards, D.J.; Buck, G.A.; Jefferson, K.K. Sequence Comparison of Vaginolysin from Different Gardnerella Species. Pathogens 2021, 10, 86. https:// doi.org/10.3390/pathogens10020086

Academic Editor: Milda Pleckaityte Received: 24 November 2020

Accepted: 14 January 2021

Published: 20 January 2021

Publisher's Note: MDPI stays neutral with regard to jurisdictional claims in published maps and institutional affiliations.

Copyright: (c) 2021 by the authors. Licensee MDPI, Basel, Switzerland. This article is an open access article distributed under the terms and conditions of the Creative Commons Attribution (CC BY) license (https:// creativecommons.org/licenses/by/ $4.0 /)$.

\begin{abstract}
Gardnerella vaginalis has recently been split into 13 distinct species. In this study, we tested the hypotheses that species-specific variations in the vaginolysin (VLY) amino acid sequence could influence the interaction between the toxin and vaginal epithelial cells and that VLY variation may be one factor that distinguishes less virulent or commensal strains from more virulent strains. This was assessed by bioinformatic analyses of publicly available Gardnerella spp. sequences and quantification of cytotoxicity and cytokine production from purified, recombinantly produced versions of VLY. After identifying conserved differences that could distinguish distinct VLY types, we analyzed metagenomic data from a cohort of female subjects from the Vaginal Human Microbiome Project to investigate whether these different VLY types exhibited any significant associations with symptoms or Gardnerella spp.-relative abundance in vaginal swab samples. While Type 1 VLY was most prevalent among the subjects and may be associated with increased reports of symptoms, subjects with Type 2 VLY dominant profiles exhibited increased relative Gardnerella spp. abundance. Our findings suggest that amino acid differences alter the interaction of VLY with vaginal keratinocytes, which may potentiate differences in bacterial vaginosis (BV) immunopathology in vivo.
\end{abstract}

Keywords: Gardnerella; vaginal microbiota; bacterial vaginosis; virulence factors; vaginolysin

\section{Introduction}

Whole-genome sequencing and phylogenetic analysis of multiple strains have resulted in the recent taxonomic re-distribution of Gardnerella vaginalis, initially described in the late 1950s [1], into 13 distinct species [2]. Vaginolysin (VLY), which is produced by most Gardnerella spp., is a cholesterol-dependent cytolysin (CDC) that may play a role as a virulence factor $[3,4]$. Previous studies suggest that in vitro expression and secretion of VLY may vary among strains. Specifically, one report found that strains isolated from subjects with clinically defined bacterial vaginosis (BV) expressed two-fold more vly compared to strains isolated from those without clinically defined BV [5]. Two additional studies found significant differences in the level of VLY secreted from different Gardnerella strains, but these differences did not correlate with the genotype [6,7]. Like other CDCs, VLY requires adequate membrane cholesterol levels for oligomerization and subsequent pore formation [3,8]. However, VLY also exhibits a requirement for the human complement receptor, $\mathrm{CD} 59$, in that the amount of toxin needed to elicit the same amount of cytotoxicity on cells lacking human CD59 is increased by 1000-fold [8]. Like other CD59-binding CDCs, 
VLY has a proline residue instead of a tryptophan in its C-terminal undecapeptide domain, a motif conserved among CDCs that is involved with membrane interaction [9]. A region proximal to the undecapeptide motif was implicated in CD59 binding in the closely related intermedilysin (ILY) [10], but crystallographic analysis of VLY and ILY implicated different residues in CD59 binding [11]. VLY is toxic to a variety of cell types, including erythrocytes, epithelial cells, and neutrophils [3,4,12]. Interestingly, however, we recently reported that the apical face of a three-dimensional tissue model of human vaginal epithelium is relatively resistant to VLY, and our data also showed that there was reduced expression of CD59 on the apical side [13]. This finding seems to contradict in vivo evidence suggesting that Gardnerella spp. and VLY are pro-inflammatory and contribute to vaginal tissue damage in BV [14-18]. Multiple reports have suggested that both commensal and BV-associated strains of Gardnerella exist, and a recent study using high-resolution bioinformatics to enable taxonomic assignment to the subspecies level found that the association of the Gardnerella genus with preterm birth is attributable to a singular subspecies clade [19-23].

In an effort to reconcile the apparent contradiction between in vivo data that VLY elicits damage and our in vitro findings that VLY largely spares the lumenal side of the vaginal epithelium, we tested the hypotheses that species-specific variations in the VLY amino acid sequence could influence the interaction between the toxin and vaginal epithelial cells and that VLY variation may be one factor that distinguishes less virulent or commensal strains from more virulent strains. While studies have noted the high amino acid identity shared by VLYs from diverse Gardnerella strains (90-99\%), there has yet to be a more comprehensive analysis of the level of VLY conservation among the now 91 publicly available Gardnerella genomes [6,19]. However, in a more recent analysis of 32 VLY amino acid sequences, one study reported a valine-to-alanine substitution variant within the undecapeptide region [24]. Comparisons among the VLYs of the recently proposed 13 distinct Gardnerella genomic species [2], however, have yet to be made. To determine whether VLY amino acid differences among the newly proposed species have functional consequences that may affect virulence, we analyzed VLY amino acid conservation among the publicly available Gardnerella genome sequences. After identifying conserved differences that could distinguish distinct VLY types, we produced recombinant toxins to test whether two distinct types affected vaginal epithelial cells differently, focusing on cytotoxicity and cytokine production. Finally, using metagenomic data from a cohort of female subjects from the Vaginal Human Microbiome Project [25] at Virginia Commonwealth University, we investigated whether these different VLY types exhibited any significant associations with symptoms reported by the subjects or the relative abundance of Gardnerella spp. in vaginal swab samples [26]. Our findings suggest that amino acid differences alter the interaction of VLY with vaginal keratinocytes, which may potentiate differences in BV immunopathology in vivo. These differences may determine whether or not a productive immune response is mounted and may explain why some but not all people with BV-like vaginal microbiome profiles experience symptoms.

\section{Results}

\subsection{Amino Acid Sequence Differences Distinguish Five Vaginolysin Types}

Alignment of 91 publicly available VLY sequences showed that the pairwise percent identities ranged from $90.48-100.0 \%$ with an average of 96.97\% (Supplementary Figure S1). Transmembrane domains and residues that were identified to participate in CD59 binding by X-ray crystallography [11] exhibited $100 \%$ identity between all VLY sequences. Domain 4 , containing the undecapeptide, proximal CD59 region, and loop regions conserved in CDCs [10,27], was highly conserved, but contained a limited number of amino acid variants (Supplementary Figure S2). Alignment of the undecapeptide and proximal CD59 regions identified five distinct vaginolysin types (Figure 1a). The undecapeptide and CD59 regions from inerolysin, a CDC produced by Lactobacillus iners [28], is shown for comparison. The VLY types were first distinguished by the sequence differences in the predicted CD59 region and then further sub-grouped based on sequence differences in the undecapeptide region. Type 1 VLY con- 
tained aspartate and serine in positions 4 and 6 of the CD59 region, respectively. Type 1A was characterized by valines at positions -1 and 6 of the undecapeptide region, while Type 1B had valine and alanine, and Type $1 \mathrm{C}$ had glutamine and valine at these positions. The CD59 region of Type 2 VLY contained asparagine and threonine at positions 4 and 6 . The undecapeptide region of this type was characterized by glutamate at position -1 and valine at position 6 . As there was no variability in this region, Type 2 was not further sub-classified. Finally, Type 3 VLY was characterized by asparagine and serine within the CD59 region and valine and leucine at positions -1 and 6 of the undecapeptide region. Types $1 \mathrm{~A}$ and $1 \mathrm{~B}$ were the most common, comprising $37.4 \%$ and $27.5 \%$ of the analyzed strains, respectively. The other types were less common: $17.6 \%$ were Type 2 and Types 1C, and three were each only represented by a single strain. In sum, this survey of VLY amino acid sequences from Gardnerella spp. isolates reveals heterogeneity within the regions of the toxin known to interact with host cells.

To determine whether these different versions of the toxin are encoded by specific Gardnerella species, we phylogenetically mapped each available strain using cpn60 gene sequences, grouped them into one of the 4 clades [29] and 13 proposed Gardnerella genomic species [2], and compared them by VLY type (Figure 1b). Type 1 VLY dominated in species 1 (G. vaginalis), 2, 3, 7, 8, 9, 10, and 13, whereas Type 2 was detected exclusively in species 5 (Gardnerella leopoldii) and 6 (Gardnerella swidsinskii), and Type 3 was only found in species 12. Species 4 (Gardnerella piotii) had a single representative strain with the Type 1 VLY, but the gene was absent in the remaining eight strains in this group, and it was absent in the single representative strain from species 11. A few other strains from species 1-3 also lacked VLY. Thus, it appears that different Gardnerella species tend to encode a particular VLY type, and some species tend to lack the vly gene altogether.

\subsection{Cytokine Secretion Profiles of VK2 Cells Challenged with Type $1 A$ and Type 2 VLY Are Similar}

While there appeared to be five distinct VLY types based on sequence differences, we suspected that some differences had a greater potential to alter protein function than others. For example, the amino acids distinguishing Type $1 \mathrm{~A}$ and $1 \mathrm{~B}$ toxins are of the same amino acid class (nonpolar aliphatic), and we therefore anticipated that they would be phenotypically similar. Type 2 VLY, on the other hand, is distinguished from Type 1 VLY by the change of a polar, uncharged asparagine residue in the CD59 region to a negatively charged aspartate residue. It also has a negatively charged glutamate residue upstream of the undecapeptide region that is replaced with a nonpolar, aliphatic valine in Type $1 \mathrm{~A}$ and Type 1B VLY. While Type 1C and Type 3 toxins exhibited sequence differences that could potentially impart functional diversity, their rarity among available Gardnerella strains prompted us to limit our in vitro analyses to Type 1A and Type 2 toxins. We hypothesized that differences in these proteins could result in differential interactions with vaginal epithelial cells and tested this hypothesis by quantifying their cytokine responses and cytotoxic potential. We cloned the vly genes from strains harboring Type 1A (G. vaginalis ATCC 14018) or Type 2 (G. leopoldii AMD) into an expression vector, expressed the proteins in E. coli, and purified them. Purified recombinant VLY (rVLY) was quantified by Bradford assay and assayed for purity by SDS PAGE (Supplementary Figure S3).

We assessed levels of cytokines secreted by VK2 cells into the media after $19 \mathrm{~h}$ of incubation with Type 1A or Type 2 rVLY (Figure 2). After incubation, cells treated with Type 1A exhibited approximately 50\% lysis, whereas cells treated with Type 2 exhibited approximately 30\% lysis. For cytokines secreted at levels above $10 \mathrm{pg} / \mathrm{mL}$, those that differed from controls by $\geq 2$-fold following rVLY exposure included IL-5, IL-7, PDGFbb, VEGF, IL-1ra, IL-6, IL-8, IL-9, IL-15, TNF- $\alpha$, GCSF, MIP-1b, and IFN- $\gamma$. VEGF was the only cytokine that exhibited a lower concentration following rVLY exposure (6.7fold). Concentrations of many cytokines were statistically different $(p<0.05)$ depending on the rVLY type added to the VK2 cells, but only IL-8 exhibited a difference $\geq 2$-fold (Figure 2, bottom). VK2 cells challenged with Type 1A rVLY secreted 4.4-fold less IL-8 than cells challenged with Type 2 rVLY. Thus, both rVLY types elicited similar cytokine secretion profiles from VK2 cell monolayers, but there were some differences in the levels 
of cytokines secreted in response to treatment with either rVLY type. As the two toxins caused significantly different levels of cell permeabilization, these differences in cytokine levels could be due to differences in lysis.

(a)

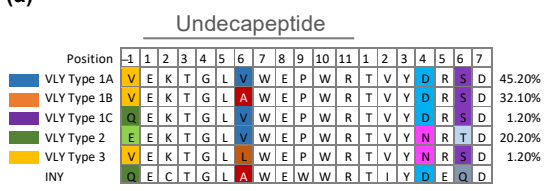

(b)
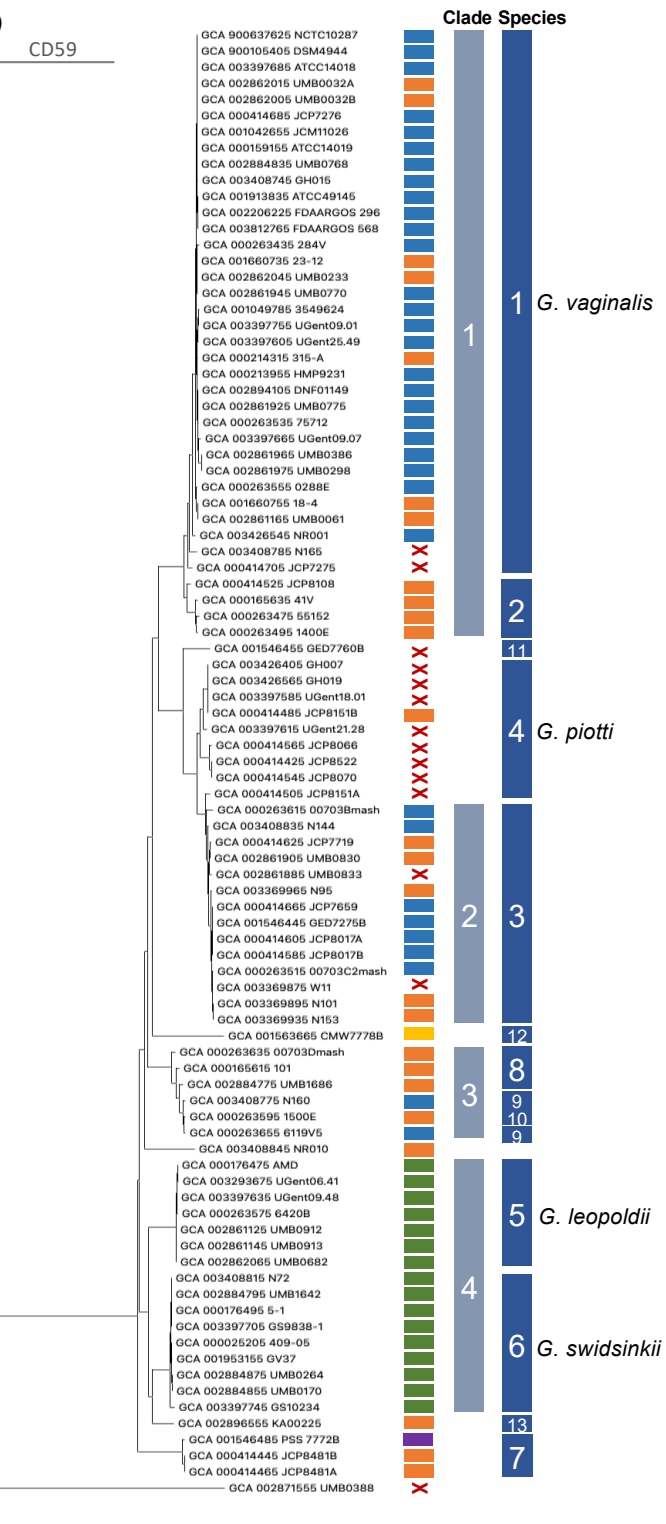

Figure 1. Five VLY types are distinguishable among Gardnerella strains. (a) VLY-type classification based on undecapeptide and CD59 region sequences of 94 Gardnerella spp. strains. Percentage values are the number of strains with a given VLY type out of all 94 strains examined. INY is the undecapeptide region from the Lactobacillus iners toxin, inerolysin. (b) Distribution of VLY types amongst Gardnerella genomic species. Neighbor-joining phylogenetic tree of full length cpn60 nucleotide sequences (1629 bp) of 91 Gardnerella spp. available genomes from GenBank). VLY types are color-coded and shown in panel (a). A red " $X$ " indicates that $v l y$ is absent. Clade distribution as described by Ahmed et al. [29] and species distribution as described in Vaneechoutte et al. [2]. 


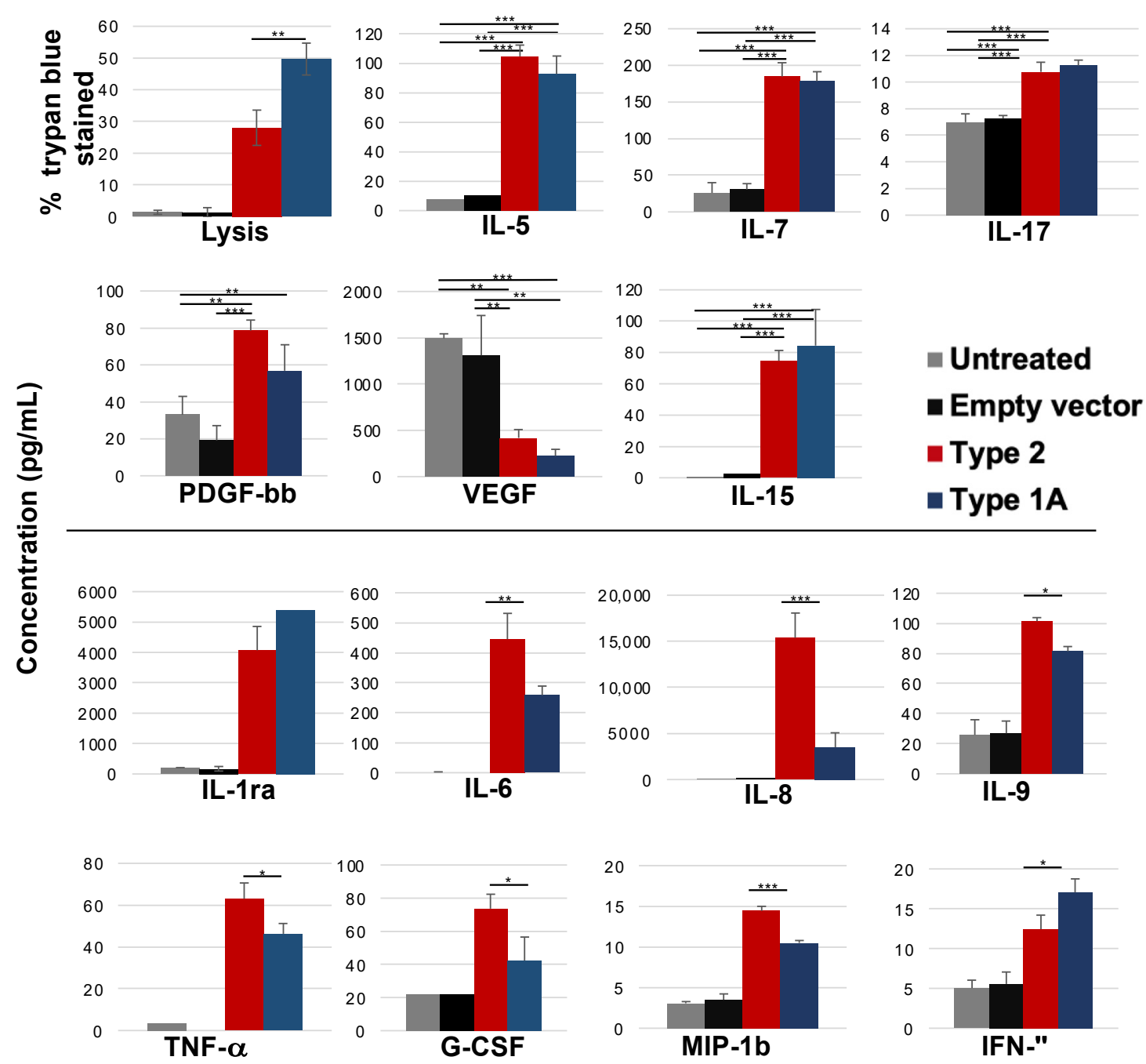

Figure 2. Effects of VLY types 1A and 2 on cytokine secretion. VK2 monolayers cultured in 24-well plates were treated with $1 \mu \mathrm{g}$ VLY $/ \mathrm{mL}$ for $19 \mathrm{~h}$. Samples of spent media from the cells were then analyzed by a multiplex cytokine bead array. For cytokines secreted at levels $\geq 10 \mathrm{pg} / \mathrm{mL}$, mean cytokine concentrations (pg/mL) exhibiting significantly different concentrations between untreated and VLY treated cells (top) or between type 2 and type 1A VLY (bottom) are shown. Error bars represent standard deviations. ${ }^{*}=p \leq 0.05,{ }^{* *} \leq 0.01,{ }^{* * *} \leq 0.001(n \geq 3)$. Protein purified from Escherichia coli containing the empty expression vector was included as a control for endotoxin or other contaminants.

\subsection{Type 1A rVLY Exhibits Greater Cytotoxicity on Cells with Less Membrane CD59}

To quantify and compare cytotoxicity, we added 1 or $0.1 \mu \mathrm{g} / \mathrm{mL}$ of Type 1A or Type 2 rVLY to VK2 cell monolayers and stained cells with trypan blue after $4 \mathrm{~h}$ of rVLY exposure. The percentage of VK2 cells that stained with trypan blue did not differ significantly between the two treatment groups (Figure 3). However, when we pre-treated cells with anti-CD59 antibody for one hour prior to rVLY challenge, we observed that the reduction in cytotoxicity was significantly greater in Type 2 rVLY-challenged cells at both toxin concentrations (Figure $3 b$ ), suggesting that rVLY Type 1A may have a higher affinity for, or depend less upon, the CD59 receptor. 


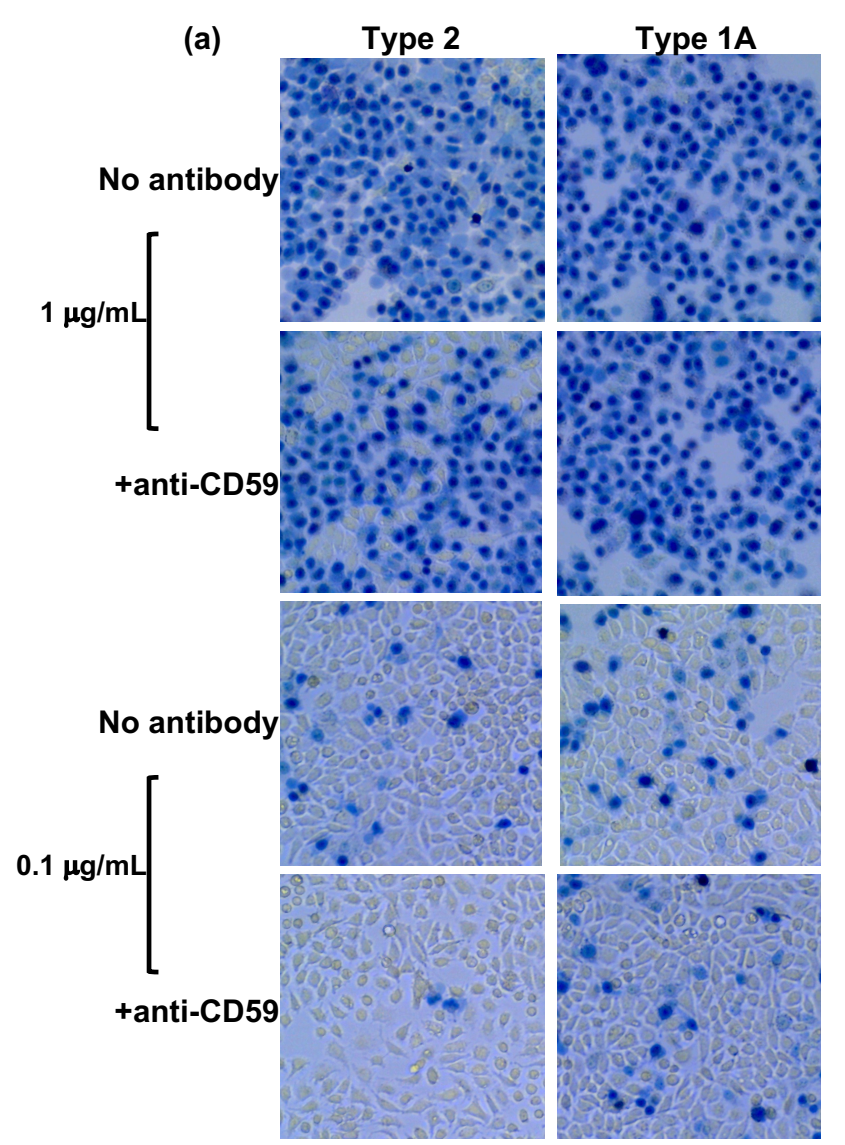

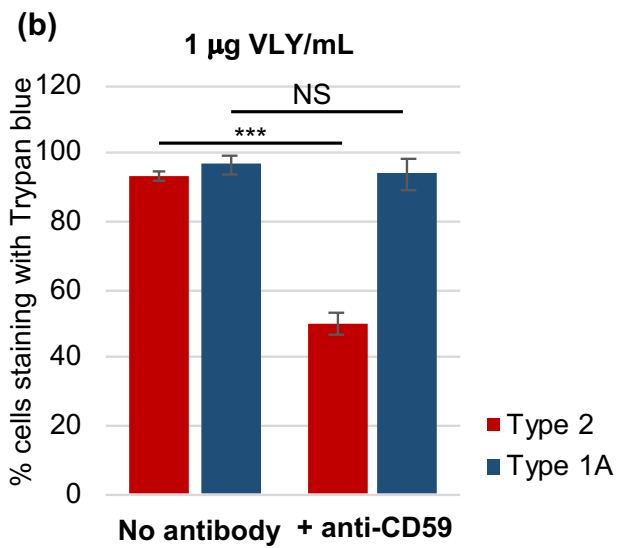

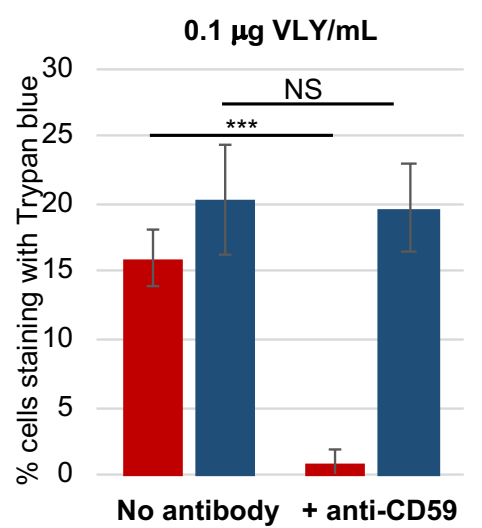

Figure 3. Anti-CD59 pre-treatment has a greater impact on Type 2 rVLY cytotoxicity. VK2 monolayers (4 days post-seeding) with and without pretreatment $(0.2 \mathrm{mg} / \mathrm{mL}$ anti-CD59 antibody for one hour) were challenged with $1 \mu \mathrm{g}$ or $0.1 \mu \mathrm{g}$ VLY $/ \mathrm{mL}$, for $4 \mathrm{~h}$. Cells were then rinsed, stained with trypan blue, and imaged. (a) Images of trypan-blue-stained cells. (b) Percentage of cells out of 1000 counted that stained with trypan blue. Assays were performed in technical and biologic triplicate and error bars represent standard deviations. NS = not significant; ${ }^{* * *}, p \leq 0.001(n \geq 3)$.

\subsection{VaHMP Study Participants Are Simultaneously Colonized by Several Gardnerella Species}

To investigate whether Gardnerella species identity and/or VLY type were associated with differences in proportional abundance within the vaginal microbiome or with differences in symptom presentation, we analyzed metagenomic data from a cohort of 62 female subjects from the Vaginal Human Microbiome Project [25] who had a positive diagnosis for BV and were vaginally colonized by Gardnerella (Supplementary Figure S4a). Since each functional VLY type is restricted to select Gardnerella species, we began our analyses by investigating the distribution of each Gardnerella species within our cohort, using cpn60 universal target sequences to resolve distinct species (Supplementary Figure S5b) [29]. Seven of the 13 defined species were present (at least 10 reads) in at least half of the samples. These included G. vaginalis, G. swidsinskii, G. piotii, and G. leopoldii in addition to species 2, 8, and 9 (Figure 4a). G. swidsinskii (20.0\%), G. vaginalis $(15.58 \%)$, species $12(9.91 \%)$, and species $9(9.34 \%)$ were the most abundant as measured by mean abundance relative to all Gardnerella species across all samples (Figure $4 \mathrm{~b}$ ). Interestingly, a majority of our participants were simultaneously colonized by multiple Gardnerella species (Figure 4c). Most subjects $(90.2 \%)$ had three or more species present in their samples, and the average number of species present per participant was nine. The abundances of each VLY type relative to all vly reads within each sample are shown in Figure $4 \mathrm{~d}$. Genomes were assembled from the metagenomic data to compare VLY amino acid sequences in our dataset to those that are publicly available. We did not detect any additional amino acid variants beyond those described above. 


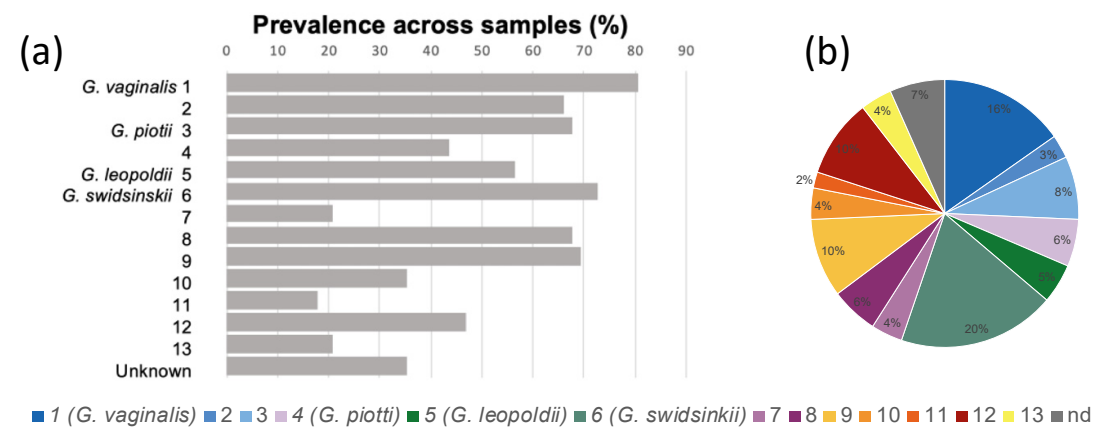

(c)

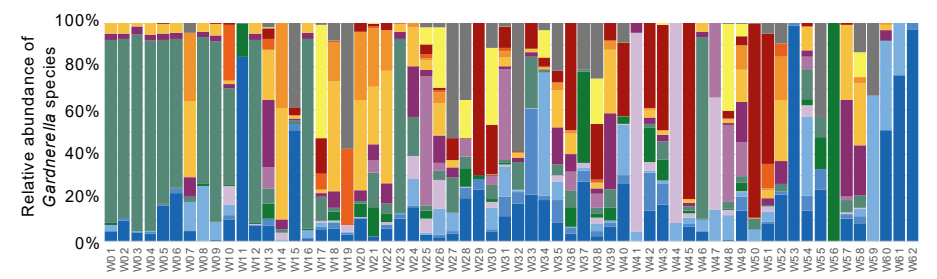

(d)

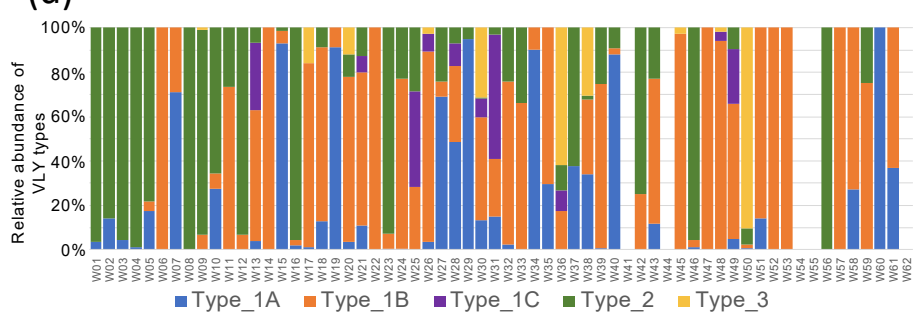

Figure 4. BV-positive subjects are simultaneously colonized by multiple Gardnerella spp. cpn60 reads were used to distinguish each Gardnerella genomic species. Each species is numbered according to the scheme reported in Vaneechoutte et al. 2019, and, where possible, labeled with a species designation; nd $=$ not determined. (a) Percentage of participants in the cohort who were colonized by each of the 13 Gardnerella species. Presence of Gardnerella species is defined as whether the number of reads mapped to the reference is 10 or higher. "Unknown", Gardnerella strain not yet classified. (b) Mean relative abundance of each genomic species across all samples. (c) Abundance of each Gardnerella species relative to the total Gardnerella population within each sample. Each bar represents one sample. Legend as indicated in panel (b). (d) Abundance of each VLY type relative to all vly reads (based on whole metagenome sequencing data) in each sample.

\subsection{Gardnerella with Type 2 Vly Gene Reach Higher Proportional Abundance}

We next assessed the distribution of VLY Types 1,2, and 3 among the cohort as a whole. We did this first by calculating the number of reads of each type across all samples and dividing by the total number of all VLY type reads from all samples. Type 1 VLY represented $49.5 \%$ of the total vly reads, while Type 2 represented $40.0 \%$ and Type 3 only $11 \%$ (Figure $5 \mathrm{a}$ ). We then grouped participants by their dominant VLY type and examined Gardnerella relative abundance and symptom frequency. If a similar number of reads were observed for two or more toxin types in a sample, that sample was classified as being dominated by both types. CLARK-S [30] was used to classify taxa from metagenomic reads and to determine the relative abundance of the taxa comprising the vaginal community profile in each subject. In contrast to what we observed for vly read distribution, participants in the Type 1 VLY group exhibited a median relative Gardnerella abundance (relative to the whole microbiome of the individual subject) of only $24.1 \%$, while those in the Type 2 VLY group exhibited a significantly higher Gardnerella abundance of $62.0 \%(p=0.0077)$ (Figure 5b). Subjects in the Type 3 VLY group exhibited the lowest Gardnerella median relative abundance at $12.9 \%(p=0.03)$. Thus, Gardnerella that encode VLY Type 2 tend to exhibit higher proportional abundance than Gardnerella strains encoding other VLY types. 
(a)

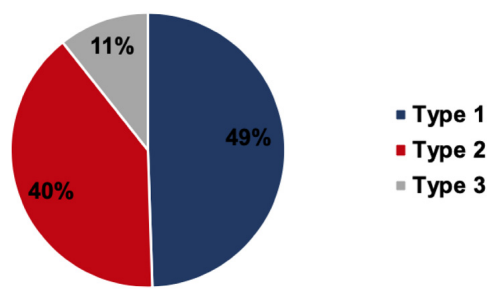

(b)

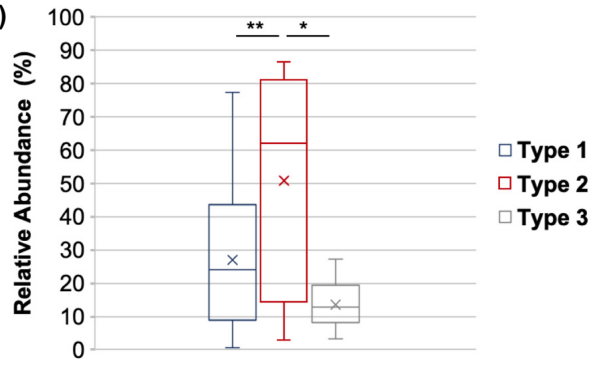

Figure 5. Relative abundance of different VLY Types. (a) The percent of each VLY type detected within the total vly reads for the entire cohort. Type 1 includes 1A, 1B, and 1C. (b) Median relative Gardnerella abundance in each VLY type group. " $x$ " marks the mean relative abundance. ${ }^{*}=p \leq 0.05,{ }^{* *} \leq 0.01$.

\subsection{Vaginal Symptoms May Be Associated with VLY Type}

Overall, $65 \%$ of the subjects reported at least one vaginal symptom (itching, odor, or discharge), while 35\% were asymptomatic (Figure 6a). After grouping participants by their VLY type, we observed that a greater number of subjects overall were dominated by Type 1 VLY $(n=39)$ compared to Types $2(n=16)$ or $3(n=7)$ (Figure $6 b)$. Interestingly, none of the Type 3 VLY-dominant subjects were asymptomatic (Figure $6 b, c)$. Subjects with Type 1 or 3 dominant profiles more frequently reported more than one symptom $(48.7 \%$ and $57.1 \%$, respectively) compared to subjects with a Type 2 dominant profile (37.5\%) (Figure 6c). Almost all reports of itching (92.9\%) were by subjects with either Type 1- or 3- dominant VLY (Figure 6c). Thus, Type 1 VLY may be more frequently associated with itching, and Type 3 may be more associated with symptoms in general.

(a)

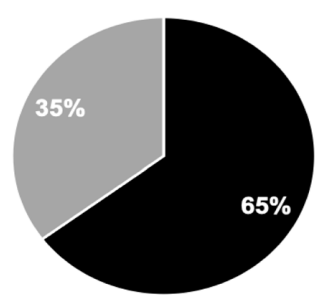

(b)

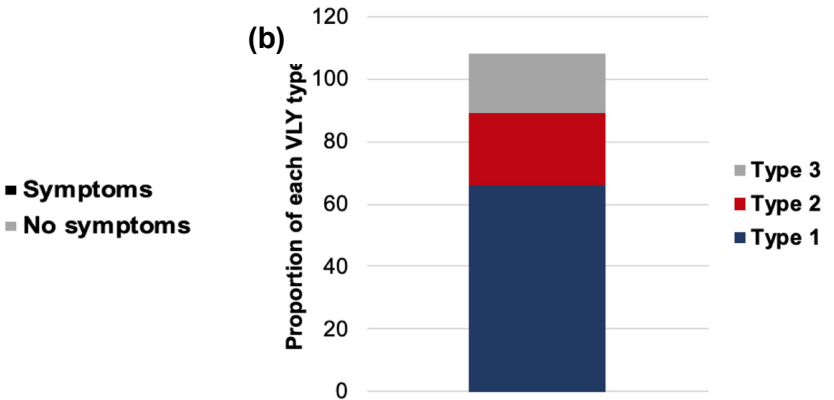

(c)
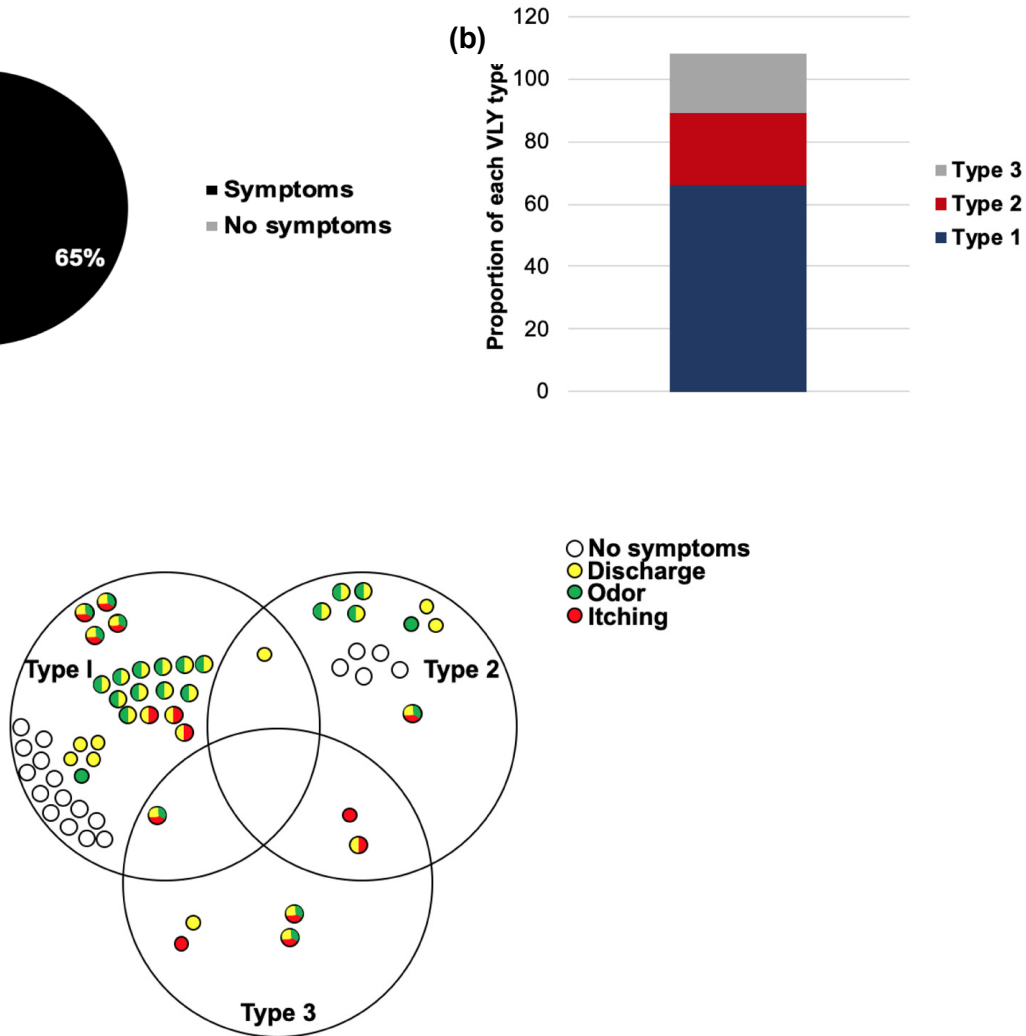

Figure 6. Subjects with type 1 VLY more frequently experience vaginal symptoms. (a) Percentage of subjects reporting vaginal symptoms. Subjects reporting "No" for each of itching, odor, or discharge were counted as "No symptoms". Subjects reporting "Not sure" $(n=2)$ were not counted. "Symptoms" represents the percentage of subjects reporting 1, 2, or 3 symptoms. (b) Proportion of each VLY type among symptomatic women. Values do not total to $100 \%$ because some samples have $>1$ dominant VLY type. (c) Symptom distribution by VLY type. 


\section{Discussion}

In this study, we compared VLY amino acid sequences across 91 publicly available Gardnerella genome sequences. Through comparisons focusing on the conserved undecapeptide and CD59 regions, we distinguished five distinct VLY types that were differentially distributed among the 13 recognized Gardnerella species. While Types $1 \mathrm{~A}-\mathrm{C}$ were found in the species groups previously defined [2] as 1-3, 7-10, and 13, VLY Type 2 was only found in species groups 5 and 6 . These distributions are consistent with a 2012 study that performed a comparative analysis of 17 Gardnerella isolates [28]. The analysis of VLY amino acid sequences of these 17 isolates provided evidence that VLY from clades 1 (species groups 1 and 2), 2 (species group 3), and 3 (species groups 8-10) engaged in recombination between clades, while clade 4 (species groups 5 and 6) retained a unique identity. Our study builds upon this by introducing VLY Type 3 as an additional candidate VLY with a unique identity. It was only identified in a single strain representing species group 12 , which does not fall into one of the four previously described clades. Inspection of the undecapeptide and proximal CD59 regions indicate characteristics of both Type 1 and Type 2 VLYs. While only $\sim 1 \%$ of the publicly available strains we analyzed encoded the Type 3 toxin, $15 \%$ of subjects from the VaHMP cohort had at least $25 \%$ of Gardnerella reads coming from this species group. Interestingly, all of these samples exhibited less than $20 \%$ relative Gardnerella abundance (relative to the whole microbiome). Thus, VLY Type 3 warrants more investigation and should be isolated and functionally compared to the other two predominant VLY types.

Alignment of Gardnerella strains based on cpn60 sequences and subsequent mapping of species group and VLY type revealed that $15 \%$ of analyzed strains were devoid of vly. While the existence of Gardnerella strains lacking the vaginolysin gene has been noted previously, we noticed that species group 4 (G. piotii) was particularly enriched in vlydeficient strains and that species group 11, represented by a single strain, was also vlydeficient $[7,30,31]$. When we looked at samples containing a large percentage (at least $25 \%$ ) of either of these two species groups among the total Gardnerella population, we observed a wide range (9.5-65\%) of relative Gardnerella abundance within the total bacterial population of each sample. We also noticed that out of all 62 samples, only one subject was colonized by a single Gardnerella species group. All other samples contained more than one species, suggesting that different species may play synergistic roles during colonization. Therefore, although several vly null strains of Gardnerella exist and may be relatively abundant during BV, this does not dispute an important role for the toxin, as VLY produced by other strains within the community could compensate. In fact, a recent publication by Bohr and colleagues provides evidence of purifying selection in VLY, suggesting that VLY is indeed important for the fitness of Gardnerella spp. [32]. Furthermore, in light of the finding that multiple Gardnerella species typically coexist in a single host, there may exist a broader interdependence between species so that infection with multiple, distinct species confers an advantage to the bacteria. Future research should be directed at defining the contributions that disparate Gardnerella species make to promote community success within the vaginal mucosa.

To assess whether amino acid differences in VLY types could alter toxin function, we performed in vitro assays using recombinantly produced toxins of the two most common divergent VLY types, Type 1A and Type 2. Similar to a 2012 study that compared the hemolytic activity of five Gardnerella strains with divergent VLY sequences [6] in which VLY sequence did not affect the hemolytic activity of VLY, we found that both Type 1A and Type 2 VLY were highly cytotoxic. However, when the vaginal epithelial cell monolayers were pre-incubated with a CD59-specific antibody to block the CD59 receptors, they were significantly more sensitive to the Type $1 \mathrm{~A}$ VLY. These results could suggest a difference in the CD59 affinity of Type 1A VLY relative to Type 2 VLY or a difference in the dependence of the two VLY types upon the co-receptor. We recently reported that in a stratified, polarized model of the human vaginal epithelium, VLY Type 2 cytotoxicity was limited to the basolateral side. Apical (lumenal) cells were resistant to the toxin [13]. 
These apical cells also exhibited reduced surface expression of CD59 relative to basolateral cells. Thus, we hypothesized that the observed differences in cytotoxicity between Type $1 \mathrm{~A}$ and Type 2 toxins could potentiate important differences in the interaction with the host epithelium in vivo. Specifically, Type 1A, if it indeed has an increased affinity for CD59, could be more damaging to the lumenal (apical) side of the vaginal mucosa, which Gardnerella spp. directly interacts with in vivo. Because of the reduced CD59 expression on this side, Type 2 VLY, if it has lower affinity for CD59, would elicit less damage. However, a crystallographic analysis of the interaction between VLY and CD59 identified different residues that participated in the bond [11], and these residues were completely conserved between all VLY types (see Supplementary Figure S1). To demonstrate that specific amino acid differences are causal in these observed differences, analysis of single amino acid substitutions within versions of the toxin will be required. Furthermore, as VLY is known to interact with cholesterol and other glycans [8,33], investigation of these molecules should be included in future studies of VLY toxin variants.

The cytokines altered in response to either toxin were, for the most part, similar. There were some significant differences in cytokine quantities between differentially challenged cells, which could be due to differences in binding, sublethal pore formation, or cell death induced by either toxin. Type 2 VLY induced a stronger IL-8 response than Type 1. If Type 1 VLY is superior at suppressing IL-8-mediated neutrophil chemotaxis, then this could further increase the damage mediated by Type 1-producing species. This could explain our finding that subjects colonized by predominantly Type 1-producing Gardnerella more frequently reported symptoms even though subjects colonized by Type 2-producing Gardnerella exhibited increased Gardnerella abundance. Type 2 VLY could promote bacterial persistence by minimizing damage and assuming a more commensal role. Our findings are in agreement with a recent publication investigating the co-occurrence patterns of the newly classified Gardnerella spp. and their associations with vaginal symptoms [29]. Hill et al. observed a strong relationship between higher relative abundance of $G$. vaginalis (Type 1 VLY) and odor and discharge symptoms. This relationship was also observed for G. swidsinskii (Type $2 \mathrm{VLY}$ ); however, the authors note that this may be due to the significant co-occurrence observed between G. vaginalis and G. swidsinskii. A similar relationship was not detected for G. leopoldii (Type 2 VLY).

In sum, this study highlights the relatively large number of Gardnerella species that often co-occur in vaginal microbiomes and reveals the relationship between species and VLY type. Future studies with versions of recombinant VLY that vary in a single amino acid and use of a model system of vaginal epithelial cells that vary in CD59 expression would greatly enhance our understanding of how the toxin functions and interacts with the host.

\section{Materials and Methods}

\subsection{Clade, Species, and VLY Type Assignment to Available Gardnerella Genomes}

In order to extract the VLY and cpn60 sequences from all the Gardnerella genomes available on NCBI, we created a profile Hidden Markov Model using HMMER3 for the pfam PF01289 (Thiol_cytolysin protein) and pfam PF00118 (Cpn60_TCP1). All proteins and coding sequences from the 110 complete and draft Gardnerella genomes available from NCBI were downloaded. Proteins were searched using HMMsearch to find hits to the PF0289 and PF00118 HMM profiles created above, and the corresponding coding sequences were extracted from each of the genomes. The Gardnerella strains from which VLY sequences were extracted (91 strains after removing duplicates) were automatically aligned using MUSCLE [34] and the final alignment was manually inspected. Following a similar process, 91 cpn60 sequences were extracted. The Neighbor-Joining tree using Kimura-2-Parameter algorithm was inferred by using the Phylogeny.fr tool $[35,36]$. Clade and species designations were assigned manually using schema outlined in Ahmed et al. 2012 and Vaneechoutte et al., 2019, respectively [2,37]. VLY type was assigned based on the scheme described below. 


\subsection{VLY Alignment}

Full-length VLY sequences were aligned using Clustal Omega and colored to highlight conserved and divergent sequences [37].

\subsection{Distinction of VLY Types}

Based on the similarities and differences observed in the sequence alignment, VLY types were first classified based on sequence differences in the CD59 region [10,26]. Type 1 VLY contained aspartate and serine in positions 4 and 6 of the CD59 region, while Type 2 VLY contained asparagine and threonine and Type 3 VLY contained asparagine and serine. The toxin types were then sub-grouped based on sequence differences in the undecapeptide region. Since Types 2 and 3 were uniform in this region, there were no subgroups for these types. Type 1 , on the other hand, had either double valine (1A), valine and alanine (1B), or glutamine and valine $(1 \mathrm{C})$ at positions -1 and 6 of the undecapeptide region.

\subsection{Expression and Purification of Recombinant VLY}

Nucleotide sequences for $v l y$ were extracted from whole-genome sequences (ATCC 14,018 GenBank assembly accession: GCA_003397685.1; AMD: GCA_000176475.1) using Hidden Markov Model. The vly gene lacking the signal sequence was amplified from G. leopolidii strain AMD (Type 2) and cloned into pET32XT as previously described [38]. G. vaginalis strain 14,018 (Type 1A) was similarly amplified and cloned with the following primers: 14018_VLY_FWD (5'-GGAAGGGATCCGCTCCTTCCGCTAAGGATTCTG-3'), 14018_VLY_REV (5'- GGAAGCTCGAGTCAGTCGTTCTTTACAGTTTCAGCAAC-3'). Protein was expressed in E. coli strain BL21(DE3) CodonPlus pRIPL (Agilent technologies). One liter cultures were grown to exponential phase $\left(\mathrm{OD}_{600}=0.5\right)$ and induced with $1 \mathrm{mM}$ IPTG for $2 \mathrm{~h}$, and the bacteria were collected by centrifugation. Bacteria were lysed in a French pressure cell in 25 mL B-PER ${ }^{\mathrm{TM}}$ (Thermo Fisher Scientific, Waltham, MA, USA) containing 1 protease inhibitor tablet (EDTA-free cOmplete $^{\mathrm{TM}}$, MilliporeSigma, Burlington, MA USA), and the lysate was cleared by centrifugation and filtration. The protein (VLY) was purified by cobalt affinity chromatography (His-Pur ${ }^{\mathrm{TM}}$, Thermo Fisher Scientific) according to manufacturer instructions, eluted in $0.25 \mathrm{M}$ imidazole, and dialyzed against $1 \mathrm{X}$ phosphate-buffered saline (PBS) at $4{ }^{\circ} \mathrm{C}$ overnight. VLY in 1X PBS was then concentrated using a $30 \mathrm{~K}$ centrifugal filter. The affinity tag was left attached. Purified, concentrated protein was then sterile-filtered, and aliquots frozen at $-80^{\circ} \mathrm{C}$ until needed. The empty vector control was similarly prepared.

\subsection{Comparison of VLY Monolayer Cytotoxicity}

VK2/E6E7 cells [39] were obtained from ATCC and cultured in complete keratinocyte medium (serum-free, $0.1 \mathrm{ng} / \mathrm{mL}$ human recombinant epidermal growth factor (EGF), $0.05 \mathrm{mg} / \mathrm{mL}$ bovine pituitary extract, $0.4 \mathrm{mM} \mathrm{CaCl}_{2}$,). Preparations of either Type $1 \mathrm{~A} \mathrm{rVLY}$ (from strain 14018) or Type 2 rVLY (from strain AMD) containing similar concentrations of the toxin, as determined by visualization of $\sim 73 \mathrm{kDa}$ band (57 kDa VLY plus $6 \mathrm{xHis}$ tag) on a NuPAGE 4 to $12 \%$ Bis-Tris gel under denaturing conditions, were compared. Protein preparations were diluted to $1 \mu \mathrm{g} / \mathrm{mL}$ in VK2 culture medium without antibiotics, and endotoxin levels were assayed using the ToxinSensor ${ }^{\mathrm{TM}}$ gel clot endotoxin assay kit. This kit detects endotoxin levels as low as $0.25 \mathrm{EU}$. The rVLY and empty vector preparations used in these assays contained undetectable levels of endotoxin. One hundred microliters of the diluted rVLY, empty vector control, or medium alone was added to approximately $3 \times 10^{4}$ adherent VK2 cells in each of 3 individual wells of 96-well plates. After $4 \mathrm{~h}$ of co-incubation at $37^{\circ} \mathrm{C}$, the media were removed and $100 \mu l$ of a $1: 1$ solution of $0.4 \%$ trypan blue and PBS was added to each well and incubated at $37^{\circ} \mathrm{C}$ for $1 \mathrm{~min}$. The trypan blue was removed, and the monolayers were imaged by light microscopy. To assess the percentage of cells staining with trypan blue, 1000 cells were counted for each sample. Some monolayers were pretreated with 1:1000 anti-CD59 (Novus Biologicals, St. Charles, MO, USA) in cell culture medium for $1 \mathrm{~h}$. Percentage trypan blue staining was calculated by diving the 
number of blue-stained cells in an image by the total number of cells and multiplying by 100. The means were compared by using a Student's t-test.

\subsection{VK2 Monolayer Cytokine Analysis}

Five hundred microliters of $1 \mu \mathrm{g} / \mathrm{mL}$ either Type $1 \mathrm{~A}$ or Type $2 \mathrm{rVLY}$ in complete keratinocyte medium were added to approximately $4 \times 10^{5}$ adherent VK2 cells per well of 24-well plates in triplicate and then incubated aerobically in $5 \% \mathrm{CO}_{2}$ at $37^{\circ} \mathrm{C}$. After $19 \mathrm{~h}$ of co-incubation, media were collected from the wells, and any cells were removed by centrifugation. Spent medium was analyzed for relative cytokine concentrations using a Bio-Plex Pro Human 27-Plex Assay. Assays were performed according to manufacturer's instructions, and plates were analyzed by the Bio-Plex MAGPIX Multiplex Reader (BIORAD, Hecules, CA, USA). Cytokine concentrations were quantified by comparing sample values to a standard curve. As a default, the standard curve of each cytokine was fit with a 5-parameter logistic model. For each cytokine, kernel density estimation (with default bandwidth parameter in JMP 15) was used to fit a smooth distribution to the relative concentrations. After the data were smoothed, the 95th percentile of the fitted distribution was used to impute out-of-range above $(\mathrm{OOR}>)$ values and the 5 th percentile was used for out-of-range below $(\mathrm{OOR}<)$ values. If the 5 th percentile was less than zero, then zero was used as the imputed value.

While 27 cytokines were assayed, only those with levels $>10 \mathrm{pg} / \mathrm{mL}$ under at least one condition are presented in Figure 2. Means were compared using a one-way ANOVA with a post hoc Tukey HSD test.

\subsection{VLY Type Metagenomic Data Analysis}

We analyzed 64 samples from the Vaginal Human Microbiome Project (VaHMP) cohort [26]. In this study, we collected more than 40,000 vaginal, cervical, introital and buccal swab samples cross-sectionally from a racially diverse cohort of 3815 female subjects visiting VCU Medical Center. Samples were collected with BD BBL CultureSwab EZ swabs. Swabs were processed immediately using MoBio Powersoil kit as described by the manufacturer. DNA samples were stored at $-80{ }^{\circ} \mathrm{C}$. DNA libraries were prepared using Illumina Truseq DNA library prep kit and sequenced on our Illumina HiSeq 2000 $\left(2 \times 100 \mathrm{~b}\right.$ PE). Samples were sequenced individually in each lane, and $5 \times 10^{8}$ paired-end reads were obtained per sample. For whole shotgun metagenomic data pre-processing, raw sequence data were demultiplexed into sample-specific fastq files using $b c l 2 f a s t q$ conversion software from Illumina. The pre-processing of raw paired-end sequence data was performed using Trimmomatic, by removing Illumina specific adapters from the reads and trimming the $5^{\prime}$ end for quality using a sliding-window approach (average Q20 over a window of $4 \mathrm{~b}$ ). Reads shorter than $70 \mathrm{bp}$ after trimming were discarded. This was followed by removal of contaminant reads of vector and human origin by aligning against the UniVec database (https:/ / www.ncbi.nlm.nih.gov/tools/vecscreen/univec/) and the hg19 build of the human genome using bwa. Two samples had fewer than 100,000 high-quality reads and were removed from further downstream analysis. Taxonomic classification of the metagenomics sequence data was performed on high-quality nonhuman reads by alignment to a custom database of bacterial genomes using CLARK-S, a discriminative space k-mer-based approach. Bacterial genomes were downloaded from the NCBI Reference Sequence Database, accessed in March 2017, supplemented with our inhouse genome assembly of Lachnospiraceae_BVAB1. Species abundances were estimated by normalizing the assigned read counts to species genome size. To determine whether variants in addition to those in the NCBI sequence data occurred in the metagenomic dataset, high-quality reads were assembled with SPAdes ver 3.8.0 [40] using the "-meta" option. Contig sequences were annotated using Prokka [41]. 


\subsection{Gardnerella Spp. Cpn60 Database}

In order to create a comprehensive cpn60 database, we first created a profile Hidden Markov Model using HMMER3 for the pfam PF00118 protein family (TCP-1/cpn60 chaperonin family). This method uses a homology detection approach by comparing sequence reads to HMM profile. All proteins and coding sequences from complete and draft from 105 Gardnerella genomes available from NCBI were downloaded. Proteins were searched using HMMsearch to find hits to the PF00118 model created above, and the corresponding coding sequences were extracted from each of the genomes. This cpn60 database was used to taxonomically classify the Gardnerella reads from the metagenomic data to the species/strain levels.

\subsection{VLY Type Distribution among Study Participants}

The number of vly reads of each type in a given sample was used to determine the "dominant" VLY type of the sample. The VLY type exhibiting a clear majority of the total vly reads for a sample (representing $\geq 50 \%$ of all vly reads) was designated as the dominant type. If two VLY types were equally represented and together made up a majority of the total vly reads in a sample, this sample was said to be dominated by both types and placed into both VLY type groups. The number of reads of a given VLY type across all samples was divided by the total number of vly reads across all samples and multiplied by 100 to give a percent value. Of the 62 samples classified as having at least 100,000 high-quality reads, two lacked any vly reads and were removed from further downstream analysis.

\subsection{Relative Bacterial Abundance in Subjects with Different VLY Types}

16S rRNA sequencing reads were used to determine the relative abundance of different bacterial species in each sample. After sample grouping by VLY type, the median relative abundance of each bacterial species was calculated. As relative abundance data were not normally distributed, a non-parametric Wilcoxon test was used to test for statistically significant differences in the median relative abundance values between VLY type groups.

\subsection{Statistical Analysis}

All data were assessed for normality by inspection of a Normal Quantile Plot. Equality of variance was assessed using the Brown-Forsythe test. The tests used for the comparison of mean or median values are described in each relevant section. All statistical analyses were performed using JMP Pro 14.0 software (SAS Institute Inc., Cary, NC, USA). $p$-values of less than 0.05 were considered significant. Fold changes of 2 or higher were considered biologically relevant.

Supplementary Materials: The following are available online at https:/ /www.mdpi.com/2076-0 817/10/2/86/s1, Figure S1: Alignment of VLY amino acid sequences. Figure S2: Alignment of VLY undecapeptide and CD59 regions reveals distinct VLY types. Figure S3: Equivalent purity and concentration of Type 1A and Type 2 rVLY. Figure S4: Relative abundance of bacterial species, Gardnerella genomic species, vly types, and inerolysin in VHMP samples.

Author Contributions: Conceptualization, E.M.G., G.A.B., and K.K.J.; methodology, E.M.G., M.G.S., G.A.B., and K.K.J.; analysis, E.M.G., M.G.S., L.E., and D.J.E.; investigation, E.M.G.; Resources, M.G.S., G.A.B., and K.K.J.; data curation, L.E.; writing—original draft preparation, E.M.G.; writing—review and editing, E.M.G., M.G.S., D.J.E., K.K.J., and G.A.B.; funding acquisition, G.A.B. and K.K.J.; visualization, E.M.G. and M.G.S.; supervision, M.G.S., G.A.B., and K.K.J. All authors have read and agreed to the published version of the manuscript.

Funding: This work was supported by National Institutes of Health [grant UH2/UH3AI083263 “The Vaginal Microbiome: Disease, Genetics and the Environment; grant U54 DE023786 “A Multi—mic Analysis of the Vaginal Microbiome during Pregnancy"].

Institutional Review Board Statement: This study was reviewed and approved by the Institutional Review Board at Virginia Commonwealth University (IRB \#HM12169, IRB \#HM15527). 
Informed Consent Statement: Written informed consent was obtained for all participants and parental permission and assent was obtained for participating minors at least 15 years of age.

Data Availability Statement: Data from the VaHMP has been deposited under dbGAP Study Accession phs000256.v3.p2. Raw metatranscriptomic sequences from the MOMS-PI project are available at NCBI's controlled-access dbGaP (Study Accession: phs001523.v1.p1).

Acknowledgments: All sequencing and analysis of sequence data was performed in the Genomics Core of the Nucleic Acids Research Facilities at VCU.

Conflicts of Interest: The authors declare no conflict of interest.

\section{References}

1. Gardner, H.L.; Dukes, C.D. Haemophilus Vaginalis Vaginitis: A Newly Defined Specific Infection Previously Classified NonSpecific Vaginitis. Am. J. Obstet. Gynecol. 1955, 69, 962-976. [CrossRef]

2. Vaneechoutte, M.; Guschin, A.; Van Simaey, L.; Gansemans, Y.; Van Nieuwerburgh, F.; Cools, P. Emended Description of Gardnerella vaginalis and Description of Gardnerella leopoldii Sp. Nov., Gardnerella piotii Sp. Nov. and Gardnerella swidsinskii sp. Nov., with Delineation of 13 Genomic Species within the Genus Gardnerella. Int. J. Syst. Evol. Microbiol. 2019, 69, 679-687. [CrossRef]

3. Gelber, S.E.; Aguilar, J.L.; Lewis, K.L.T.; Ratner, A.J. Functional and Phylogenetic Characterization of Vaginolysin, the HumanSpecific Cytolysin from Gardnerella vaginalis. J. Bacteriol. 2008, 190, 3896-3903. [CrossRef]

4. Rottini, G.; Dobrina, A.; Forgiarini, O.; Nardon, E.; Amirante, G.A.; Patriarca, P. Identification and Partial Characterization of a Cytolytic Toxin Produced by Gardnerella vaginalis. Infect. Immun. 1990, 58, 3751-3758. [CrossRef]

5. Castro, J.; Alves, P.; Sousa, C.; Cereija, T.; França, Â.; Jefferson, K.K.; Cerca, N. Using an In-Vitro Biofilm Model to Assess the Virulence Potential of Bacterial Vaginosis or Non- Bacterial Vaginosis Gardnerella vaginalis Isolates. Sci. Rep. 2015, 5, 11640. [CrossRef]

6. Pleckaityte, M.; Janulaitiene, M.; Lasickiene, R.; Zvirbliene, A. Genetic and Biochemical Diversity of Gardnerella vaginalis Strains Isolated from Women with Bacterial Vaginosis. FEMS Immunol. Med. Microbiol. 2012, 65, 69-77. [CrossRef]

7. Janulaitiene, M.; Gegzna, V.; Baranauskiene, L.; Bulavaite, A.; Simanavicius, M.; Pleckaityte, M. Phenotypic Characterization of Gardnerella vaginalis Subgroups Suggests Differences in Their Virulence Potential. PLoS ONE 2018, 13, e0200625. [CrossRef]

8. Zilnyte, M.; Venclovas, C.; Zvirbliene, A.; Pleckaityte, M. The Cytolytic Activity of Vaginolysin Strictly Depends on Cholesterol and Is Potentiated by Human CD59. Toxins 2015, 7, 110-128. [CrossRef]

9. Ramachandran, R.; Heuck, A.P.; Tweten, R.K.; Johnson, A.E. Structural Insights into the Membrane-Anchoring Mechanism of a Cholesterol-Dependent Cytolysin. Nat. Struct. Biol. 2002, 9, 823-827. [CrossRef]

10. Hughes, T.R.; Ross, K.S.; Cowan, G.J.M.; Sivasankar, B.; Harris, C.L.; Mitchell, T.J.; Morgan, B.P. Identification of the High Affinity Binding Site in the Streptococcus intermedius Toxin Intermedilysin for Its Membrane Receptor, the Human Complement Regulator CD59. Mol. Immunol. 2009, 46, 1561-1567. [CrossRef]

11. Lawrence, S.L.; Gorman, M.A.; Feil, S.C.; Mulhern, T.D.; Kuiper, M.J.; Ratner, A.J.; Tweten, R.K.; Morton, C.J.; Parker, M.W. Structural Basis for Receptor Recognition by the Human CD59-Responsive Cholesterol-Dependent Cytolysins. Structure 2016, 24, 1488-1498. [CrossRef]

12. Kretzschmar, U.M.; Hammann, R.; Kutzner, H.J. Purification and Characterization of Gardnerella vaginalis Hemolysin. Curr. Microbiol. 1991, 23, 7-132. [CrossRef]

13. Garcia, E.M.; Kraskauskiene, V.; Koblinski, J.E.; Jefferson, K.K. Interaction of Gardnerella vaginalis and Vaginolysin with the Apical versus Basolateral Face of a Three-Dimensional Model of Vaginal Epithelium. Infect. Immun. 2019, 87. [CrossRef]

14. Nowak, R.G.; Randis, T.M.; Desai, P.; He, X.; Robinson, C.K.; Rath, J.; Glover, E.D.; Ratner, A.J.; Ravel, J.; Brotman, R.M. Higher Levels of a Cytotoxic Protein, Vaginolysin, in Lactobacillus-Deficient Community State Types at the Vaginal Mucosa. Sex. Transm. Dis. 2018, 45, e14-e17. [CrossRef]

15. Hedges, S.R.; Barrientes, F.; Desmond, R.A.; Schwebke, J.R. Local and Systemic Cytokine Levels in Relation to Changes in Vaginal Flora. J. Infect. Dis. 2006, 193, 556-562. [CrossRef]

16. Cauci, S.; Driussi, S.; Guaschino, S.; Isola, M.; Quadrifoglio, F. Correlation of Local Interleukin-1 $\beta$ Levels with Specific IgA Response Against Gardnerella vaginalis Cytolysin in Women with Bacterial Vaginosis. Am. J. Reprod. Immunol. 2002, 47, 257-264. [CrossRef]

17. Cauci, S.; Scrimin, F.; Driussi, S.; Ceccone, S.; Monte, R.; Fant, L.; Quadrifoglio, F. Specific Immune Response against Gardnerella vaginalis Hemolysin in Patients with Bacterial Vaginosis. Am. J. Obstet. Gynecol. 1996, 175, 1601-1605. [CrossRef]

18. Cauci, S.; Thorsen, P.; Schendel, D.E.; Bremmelgaard, A.; Quadrifoglio, F.; Guaschino, S. Determination of Immunoglobulin A against Gardnerella vaginalis Hemolysin, Sialidase, and Prolidase Activities in Vaginal Fluid: Implications for Adverse Pregnancy Outcomes. J. Clin. Microbiol. 2003, 41, 435-438. [CrossRef]

19. Harwich, M.D., Jr.; Alves, J.M.; Buck, G.A.; Strauss Iii, J.F.; Patterson, J.L.; Oki, A.T.; Girerd, P.H.; Jefferson, K.K. Drawing the Line between Commensal and Pathogenic Gardnerella vaginalis through Genome Analysis and Virulence Studies. BMC Genom. 2010, 11, 375. [CrossRef] 
20. Yeoman, C.J.; Yildirim, S.; Thomas, S.M.; Durkin, A.S.; Torralba, M.; Buhay, C.J.; Ding, Y.; Dugan-rocha, S.P.; Muzny, D.M.; Qin, X.; et al. Comparative Genomics of Gardnerella vaginalis Strains Reveals Substantial Differences in Metabolic and Virulence Potential. PLoS ONE 2010, 5, e12411. [CrossRef]

21. Cornejo, O.E.; Hickey, R.J.; Suzuki, H.; Forney, L.J. Focusing the Diversity of Gardnerella vaginalis through the Lens of Ecotypes. Evol. Appl. 2018, 11, 312-324. [CrossRef]

22. Fredricks, D.N.; Fiedler, T.L.; Thomas, K.K.; Oakley, B.B.; Marrazzo, J.M. Targeted PCR for Detection of Vaginal Bacteria Associated with Bacterial Vaginosis. J. Clin. Microbiol. 2007, 45, 3270-3276. [CrossRef]

23. Callahan, B.J.; DiGiulio, D.B.; Goltsman, D.S.A.; Sun, C.L.; Costello, E.K.; Jeganathan, P.; Biggio, J.R.; Wong, R.J.; Druzin, M.L.; Shaw, G.M.; et al. Replication and Refinement of a Vaginal Microbial Signature of Preterm Birth in Two Racially Distinct Cohorts of US Women. Proc. Natl. Acad. Sci. USA 2017, 114, 9966-9971. [CrossRef]

24. Ma, B.; France, M.T.; Crabtree, J.; Holm, J.B.; Humphrys, M.S.; Brotman, R.M.; Ravel, J. A Comprehensive Non-Redundant Gene Catalog Reveals Extensive within-Community Intraspecies Diversity in the Human Vagina. Nat. Commun. 2020, 11, 940. [CrossRef]

25. Fettweis, J.; Alves, J.; Borzelleca, J.; Brooks, J.; Friedline, C.; Gao, Y.; Gao, X.; Girerd, P.; Harwich, M.; Hendricks, S.; et al. The Vaginal Microbiome: Disease, Genetics and the Environment. Nat. Preced. 2010. [CrossRef]

26. Macklaim, J.M.; Gloor, G.B.; Anukam, K.C.; Cribby, S.; Reid, G. At the Crossroads of Vaginal Health and Disease, the Genome Sequence of Lactobacillus iners AB-1. Proc. Natl. Acad. Sci. USA 2011, 108, 4688-4695. [CrossRef]

27. Rampersaud, R.; Planet, P.J.; Randis, T.M.; Kulkarni, R.; Aguilar, J.L.; Lehrer, R.I.; Ratner, A.J. Inerolysin, a Cholesterol-Dependent Cytolysin Produced by Lactobacillus iners. J. Bacteriol. 2011, 193, 1034-1041. [CrossRef]

28. Ahmed, A.; Earl, J.; Retchless, A.; Hillier, S.L.; Rabe, L.K.; Cherpes, T.L.; Powell, E.; Janto, B.; Eutsey, R.; Luisa Hiller, N.; et al. Comparative Genomic Analyses of 17 Clinical Isolates of Gardnerella vaginalis Provide Evidence of Multiple Genetically Isolated Clades Consistent with Subspeciation into Genovars. J. Bacteriol. 2012, 194, 3922-3937. [CrossRef]

29. Hill, J.E.; Albert, A.Y.K. Resolution and Co-Occurrence Patterns of Gardnerella leopoldii, Gardnerella swidsinskii, Gardnerella piotii and Gardnerella vaginalis within the Vaginal Microbiome. Infect. Immun. 2019. [CrossRef]

30. Ounit, R.; Wanamaker, S.; Close, T.J.; Lonardi, S. CLARK: Fast and Accurate Classification of Metagenomic and Genomic Sequences Using Discriminative k-Mers. BMC Genom. 2015, 16, 1-13. [CrossRef]

31. Janulaitiene, M.; Paliulyte, V.; Grinceviciene, S.; Zakareviciene, J.; Vladisauskiene, A.; Marcinkute, A.; Pleckaityte, M. Prevalence and Distribution of Gardnerella vaginalis Subgroups in Women with and without Bacterial Vaginosis. BMC Infect. Dis. 2017, 17, 394. [CrossRef]

32. Bohr, L.L.; Mortimer, T.D.; Pepperell, C.S. Lateral Gene Transfer Shapes Diversity of Gardnerella spp. Front. Cell. Infect. Microbiol. 2020, 10, 293. [CrossRef]

33. Shewell, L.K.; Day, C.J.; Jen, F.E.-C.; Haselhorst, T.; Atack, J.M.; Reijneveld, J.F.; Everest-Dass, A.; James, D.B.A.; Boguslawski, K.M.; Brouwer, S.; et al. All Major Cholesterol-Dependent Cytolysins Use Glycans as Cellular Receptors. Sci. Adv. 2020, 6, eaaz4926. [CrossRef]

34. Edgar, R.C. MUSCLE: A Multiple Sequence Alignment Method with Reduced Time and Space Complexity. BMC Bioinform. 2004, 5, 113. [CrossRef]

35. Dereeper, A.; Guignon, V.; Blanc, G.; Audic, S.; Buffet, S.; Chevenet, F.; Dufayard, J.; Guindon, S.; Lefort, V.; Lescot, M.; et al. Phylogeny.Fr: Robust Phylogenetic Analysis for the Non-Specialist I Nucleic Acids Research I Oxford Academic. Nucleic Acids Res. 2008, 36, W465-W469. [CrossRef]

36. Dereeper, A.; Audic, S.; Claverie, J.M.; Blanc, G. BLAST-EXPLORER Helps You Building Datasets for Phylogenetic Analysis. BMC Evol. Biol. 2010, 10, 8. [CrossRef]

37. Madeira, F.; mi Park, Y.; Lee, J.; Buso, N.; Gur, T.; Madhusoodanan, N.; Basutkar, P.; Tivey, A.R.N.; Potter, S.C.; Finn, R.D.; et al. The EMBL-EBI Search and Sequence Analysis Tools APIs in 2019. Nucleic Acids Res. 2019, 47, W636-W641. [CrossRef]

38. Abdelmaksoud, A.A.; Girerd, P.H.; Garcia, E.M.; Brooks, J.P.; Leftwich, L.M.; Sheth, N.U.; Bradley, S.P.; Serrano, M.G.; Fettweis, J.M.; Huang, B.; et al. Association between Statin Use, the Vaginal Microbiome, and Gardnerella vaginalis Vaginolysin-Mediated Cytotoxicity. PLoS ONE 2017, 12, e0183765. [CrossRef]

39. Fichorova, R.N.; Rheinwald, J.G.; Anderson, D.J. Generation of Papillomavirus-Immortalized Cell Lines from Normal Human Ectocervical, Endocervical, and Vaginal Epithelium That Maintain Expression of Tissue-Specific Differentiation Proteins. Biol. Reprod. 1997, 57, 847-855. [CrossRef]

40. Bankevich, A.; Nurk, S.; Antipov, D.; Gurevich, A.A.; Dvorkin, M.; Kulikov, A.S.; Lesin, V.M.; Nikolenko, S.I.; Pham, S.; Prjibelski, A.D.; et al. SPAdes: A New Genome Assembly Algorithm and Its Applications to Single-Cell Sequencing. J. Comput. Biol. J. Comput. Mol. Cell Biol. 2012, 19, 455-477. [CrossRef]

41. Seemann, T. Prokka: Rapid Prokaryotic Genome Annotation. Bioinform. Oxf. Engl. 2014, 30, 2068-2069. [CrossRef] [PubMed] 